\title{
Measuring infection transmission in a stochastic SIV model with infection reintroduction and imperfect vaccine
}

\author{
M Gamboa · MJ Lopez-Herrero
}

Received: date / Accepted: date

\begin{abstract}
An additional compartment of vaccinated individuals is considered in a SIS stochastic epidemic model with infection reintroduction. The quantification of the spread of the disease is modeled by a continuous time Markov chain. A well-known measure of the initial transmission potential is the basic reproduction number $R_{0}$, which determines the herd immunity threshold or the critical proportion of immune individuals required to stop the spread of a disease when a vaccine offers a complete protection. Due to repeated contacts between the typical infective and previously infected individuals, $R_{0}$ overestimates the average number of secondary infections and leads to, perhaps unnecessary, high immunization coverage. Assuming that the vaccine is imperfect, alternative measures to $R_{0}$ are defined in order to study the influence of the initial coverage and vaccine efficacy on the transmission of the epidemic.
\end{abstract}

Keywords Stochastic Markovian epidemic - Imperfect vaccine · Basic reproduction number

Mathematics Subject Classification (2000) 92D30 - 60J28 $\cdot 60 \mathrm{~J} 22$

\begin{abstract}
The authors would like to thank the referees and the editor for their useful comments and suggestions, which helped us to improve the manuscript. Financial support for this work was provided by the Government of Spain (Department of Science, Technology and Innovation) and the European Commission through project MTM 2014-58091-P. The first author is grateful for the economical support of Banco Santander and Universidad Complutense of Madrid (Pre-doctoral researcher contract CT42/18-CT43/18)
\end{abstract}

M Gamboa

Faculty of Statistical Studies, Complutense University of Madrid, Spain

E-mail: mgamboa@ucm.es

MJ Lopez-Herrero (Corresponding author)

Faculty of Statistical Studies, Complutense University of Madrid, Spain

E-mail: lherrero@estad.ucm.es 


\section{Introduction}

Mathematical modeling is an essential tool to represent the progress of an epidemic through a population. It is commonly accepted that the work of Kermack and Mckendrick (1927) is the prototype of almost all epidemiological models based on a classification of individuals by their epidemic status. Since then, many other compartmental models have been developed to investigate a diverse range of communicable diseases to obtain a better knowledge of their transmission mechanisms (Anderson and Britton 2000; Kretzschmar et al. 2001; Aguiar et al. 2010; Artalejo and Lopez-Herrero 2014; Liu et al. 2018).

A common assumption is that the communicable disease spreads in a community of constant size. The population can be closed in the sense that infectious individuals can infect only other individuals within the population under study during the epidemic's time span. However, assuming reintroduction of the disease through contact with infected individuals from other areas could represent a more realistic scenario (Marchette and Wierman 2004; Stone et al. 2007; Amador 2016).

After infection, it is assumed that patients recover due to their own immune system which acts as a body's defense force against germs and other invading substances. Hence, to reduce the incidence of an infectious disease requires improving sanitary and living conditions. In that sense, we cannot ignore the impact of vaccination on this reduction, especially in the developing world. Vaccines activate the immune system's capacity of producing antibodies to fight diseases without exposing it to diseases-producing pathogens. If a vaccinated person comes into contact with the disease for which she/he has been vaccinated, her/his immune system recognizes the invading germs and immediately produces the antibodies that will kill foreign invaders. Generally, vaccines provide immunity similar to that acquired from the natural infection, and duration of protection varies depending on diseases and also on vaccine strains. Lifelong immunity is not always provided by vaccination and usually several doses of vaccine may be required. Furthermore, the immune response may wane over time and it is necessary to administrate new doses of vaccine to increase or restore immunity.

As it is an effective method of disease control, recent epidemiological models (Kribs-Zaleta and Martcheva 2002; Arino et al. 2003; Arino et al. 2010; Ball and Sirl 2013; Samanta 2015; Ball and Sirl 2018; Li and Zhang 2019) have added a vaccination compartment and vaccination strategies into their mathematical model. Some papers discussing the impact of vaccination on the spread of an epidemic assume complete protection (Iannelli et al. 2005; Alexander et al. 2006; Ball et al. 2007; Lin et al. 2014; Eckalbar and Eckalbar 2015; Guo 2017) but even vaccine efficacy, as measured by observational studies, is not $100 \%$ (e.g., measles: $90-95 \%$, mumps: $72-88 \%$ or rubella: $95-98 \%$ ) and depends on internal or individual factors, as well as on the dose and strain of the vaccine virus (Demicheli et al. 2012). In scientific literature, vaccine efficacy and effectiveness are often used interchangeably. Vaccine efficacy represents the reduction in the risk of infection at individual level under optimal condi- 
tion (e.g., randomized controlled trials) while vaccine effectiveness compares rates of the transmission of the disease between vaccinated and unvaccinated individuals once the vaccine is approved for use in the general population.

The SIS model with imperfect vaccine has been studied from a deterministic point of view in the papers by Moghadas (2004) and Safan and Rihan (2014), under nonlinear incidence in Xiao and Tang (2010) and Yang et al. (2015), and with inclusion of a latency period as well as psychological effects in both susceptible and vaccinated individuals in Cheng et al. (2015). A SIV model with stochastic perturbations is discussed in Liu et al. (2018) and López-García (2016).

The above-mentioned studies deal with large populations, but transmission patterns become quantitatively different when a small population is involved. Firstly, individual variations of infectivity, recovery periods and vaccine protection, even for a homogeneous social group, should be taken into account. Moreover, when the susceptible population becomes depleted the extent of the epidemic is interrupted and, for closed populations, the extinction of the disease is possible (Keeling and Ross 2008).

The aim of this paper is to quantify the spread of an infectious disease that does not confer immunity, within a population that is partially protected against the disease by a vaccine. A continuous time Markov chain $(C T M C)$ model represents changes in the composition of infected and vaccinated classes. Two random variables will quantify the transmission of the epidemic process with reintroduction: $R_{e 0}$, the number of infectious cases caused directly by the first infected individual, and $R_{p}$, which is the number of infectious cases caused by any infectious spreader. These random variables act as stochastic counterparts to the basic reproduction number, $R_{0}$, and more specifically the control reproduction number, $R_{c}$, when there is an available vaccine.

In the literature, the term herd-immunity threshold refers to the critical proportion of immune individuals that is needed to interrupt epidemic transmission in a population. There is a simple relationship between herd-immunity coverage and the basic reproduction number. If a perfect vaccine is available and a fraction $f$ of the population is vaccinated, then the disease will not spread if $(1-f) R_{0}<1$. In general terms, we can represent the quality of a vaccine by a measure of the vaccine imperfection $h \in[0,1]$, with $h=0$ indicating a perfect vaccine, and $h=1$ a useless vaccine. For imperfect but not useless vaccines, the critical vaccination coverage level to eradicate the infection is related to the proportion $\left(1-1 / R_{0}\right) /(1-h)$. In any case, herd immunity depends on estimates of $R_{0}$, and is the result of a reduction in viral transmission caused by removing vaccinated individuals from the susceptible class. We will investigate the spread of the disease directly, at any time, by updating the current population state, and we will apply our alternative measures to control disease spread by fixing an adequate vaccine coverage level.

The structure of the paper is as follows. In Section 2, we describe the SIV stochastic model with infection reintroduction and summarize results for its long term behavior in terms of the reintroduction parameter. Section 3 introduces the proposed random variables, $R_{e 0}$ and $R_{p}$, as measures of the in- 
fection transmission in a partially vaccinated population. Theoretical and algorithmic results will provide probability mass functions and moments for $R_{e 0}$ and $R_{p}$, depending on the initial vaccine coverage and effectiveness. Section 4 illustrates the applicability of the algorithmic results and presents several qualitative facts regarding the infection transmission. Finally, we present some concluding remarks and tentative future research lines.

\section{Model description}

We consider a homogeneous and uniformly mixing population of constant size, $N$, where individuals are affected by a contagious disease. This disease is transmitted by direct contact with an infected individual. The population is not isolated, so we assume that there is an additional source of infection due to external contacts. We suppose that some individuals in the population have been protected against the disease with an available vaccine that confers immunity, but it is not a perfect vaccine and some contacts between vaccinated and infectious individuals produce an effective contagion. Once a vaccinated individual gets the infection, he no longer belongs to the class of individuals that have been vaccinated and he belongs to the infective class while he is infectious. Recovered individuals become susceptible to the disease, no matter if they were previously vaccinated or not. Consequently, individuals in the population are classified into three separate classes, namely susceptible, vaccinated and infected. Vaccination was implemented at $t=0$ and no vaccination will take place after this epoch.

In general terms, the underlying mathematical model involves a SIV model, where movement of individuals among the three epidemiological classes is shown in Figure 1, with $S$ denoting the class of susceptible individuals, $I$ denoting the class of infected individuals, and $V$ denoting the class of vaccinated individuals.

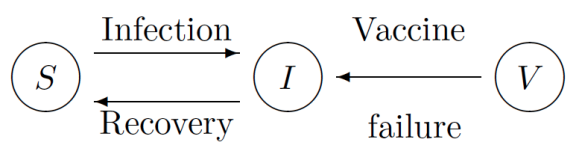

Fig. 1 SIV compartmental diagram

At any time $t>0$, the state of the epidemic is described by random variables $S(t), V(t), I(t)$, that record the number of susceptible, vaccinated and infective individuals, respectively, at time $t$. According to the constant size hypothesis we have

$$
S(t)+V(t)+I(t)=N
$$


We represent the evolution of the disease in terms of a two-dimensional CTMC: $X=\{(V(t), I(t)), t \geq 0\}$. Assuming that initially the population contains $v_{0}$ vaccinated individuals, with $0 \leq v_{0} \leq N$, the state space of $X$ is $\mathrm{S}=\{(v, i)$ : $\left.0 \leq v \leq v_{0}, 0 \leq i \leq N, 0 \leq v+i \leq N\right\}$ that contains $\left(v_{0}+1\right)\left(N+1-v_{0} / 2\right)$ states. Notice that once $v=0$ the vaccination compartment is empty and the underlying model is the standard SIS epidemic one.

Next, we describe the dynamics of the Markov chain, $X$, in full details. See Table 1 for a summary of parameters used. The exhaustive description of the events and their transition rates is presented in Table 2. Finally, a diagram showing transitions from a general state $(v, i) \in \mathrm{S}$ is depicted in Figure 2.

Table 1 Parameters of the model

\begin{tabular}{|ll|}
\hline Population size & $N$ \\
Disease internal transmission rate & $\beta$ \\
Disease external transmission rate & $\xi$ \\
Probability of vaccine failure & $h$ \\
Recovery rate & $\gamma$ \\
\hline
\end{tabular}

Table 2 Effective events and their transition rates

\begin{tabular}{lll}
\hline Effective event & Transition & Rate \\
\hline Susceptible-Infected internal contagion & $(v, i) \rightarrow(v, i+1)$ & $\beta i(N-v-i) / N$ \\
Susceptible-Infected external contagion & $(v, i) \rightarrow(v, i+1)$ & $\xi(N-v-i)$ \\
Vaccinated-Infected internal contagion & $(v, i) \rightarrow(v-1, i+1)$ & $h \beta i v / N$ \\
Vaccinated-Infected external contagion & $(v, i) \rightarrow(v-1, i+1)$ & $h \xi v$ \\
Recovery and loss of immunity & $(v, i) \rightarrow(v, i-1)$ & $\gamma i$ \\
\hline
\end{tabular}

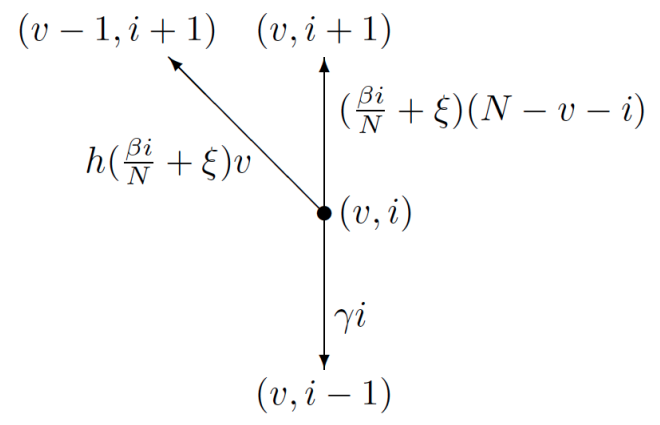

Fig. 2 Transitions among states 
To understand the dynamics of the model it is necessary to describe the events that cause a change in the current model state $(v, i)$. There are five possible effective events that are listed in Table 2 .

$E_{1}$. A susceptible individual gets the infection from an infective individual within the population.

$E_{2}$. A susceptible individual gets the infection from an external source of infection.

$E_{3}$. Due to vaccine failure, a vaccinated individual becomes infected from an infective individual within the population.

$E_{4}$. Due to vaccine failure, a vaccinated individual becomes infected from an external source of infection.

$E_{5}$. An infective individual is recovered and becomes susceptible.

Sojourn times at each state in $\mathrm{S}$ are independent and exponential random variables, with rate

$$
q_{v, i}=\left(\frac{\beta i}{N}+\xi\right)(N-v-i)+h\left(\frac{\beta i}{N}+\xi\right) v+\gamma i
$$

To describe $\mathbf{Q}$, the infinitesimal generator of the Markov chain $X$, we partition the state space in levels regarding the number of vaccinated individuals, that is $\mathrm{S}=\cup_{v=0}^{v_{0}} L(v)$, where level $L(v)=\{(v, i) \in \mathrm{S}: 0 \leq i \leq N-v\}$, for $0 \leq v \leq v_{0}$, which contains $(N+1-v)$ states. Then, we can express the infinitesimal generator of $X$ in the following non-null block form

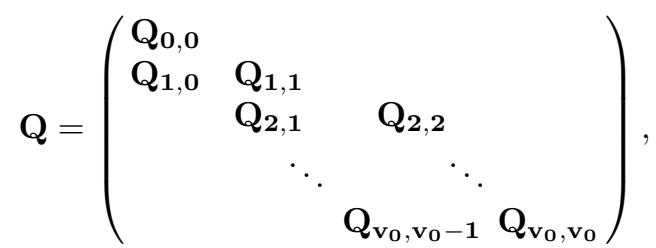

where $\mathbf{Q}_{\mathbf{v}, \mathbf{v}^{*}}$, for $0 \leq v, v^{*} \leq v_{0}$, are matrices of dimension $(N+1-v) \times(N+$ $\left.1-v^{*}\right)$. The blocks $\mathbf{Q}_{\mathbf{v}, \mathbf{v}-\mathbf{1}}$, for $1 \leq v \leq v_{0}$, correspond to transitions due to vaccine failures, and the blocks $\mathbf{Q}_{\mathbf{v}, \mathbf{v}}$, for $0 \leq v \leq v_{0}$, correspond to transitions within level $L(v)$ that are due either to infections of susceptible or recoveries. Non-null sub-matrices are described as follows

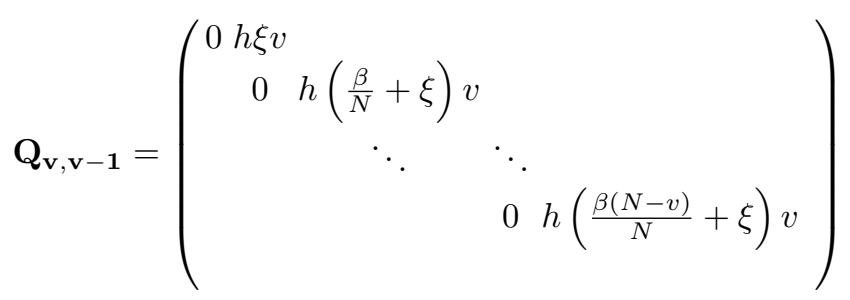


and

$$
\mathbf{Q}_{\mathbf{v}, \mathbf{v}}=\left(\begin{array}{cccc}
-q_{v, 0} & \xi(N-v) & & \\
\gamma & -q_{v, 1} & \left(\frac{\beta}{N}+\xi\right)(N-v-1) & \\
& \ddots & \ddots & \ddots \\
& (N-v-1) \gamma & -q_{v, N-v-1} & \frac{\beta(N-v-1)}{N}+\xi \\
& & (N-v) \gamma & -q_{v, N-v}
\end{array}\right)
$$

The above block bidiagonal structure of $\mathbf{Q}$ guarantees that square matrices appearing in the forthcoming theoretical results are, at most, tridiagonal and diagonally dominant. Thus, they are non-singular, and linear systems involving these matrices can be solved efficiently by a simplified form of the Gaussian elimination algorithm (Golub and van Loan 1996).

\subsection{Stationary behavior}

As we are dealing with a finite state CTMC, the long-term behavior of $X$ depends on the structure of communicating classes of absorbing states. In that sense, we notice that the reintroduction parameter (that is, the external transmission rate $\xi$ ) plays an important role in the classification of the states in S. If we assume that the population is isolated and contagions are produced only by internal contacts; i.e., $\xi=0$, then states with 0 infective individuals are absorbing. This fact and the finiteness of the state space guarantee that the process will become absorbed into any of the non-communicating classes of absorbing states with probability one. Hence, the epidemic extinction is certain and outbreaks involve a single epidemic episode that will last a finite expected time.

On the other hand, when $\xi>0$, the state space of the finite CTMC $X$ contains a single absorbing set given by $L(0)=\{(0, i): 0 \leq i \leq N\}$. Thus, once the process enters into $L(0)$ it can move across these states but it can not leave the absorbing set. Hence the stationary distribution assigns mass to every state with no vaccinated individuals. Which means that, in the model with external source of infection, occasionally the disease is faded away (i.e., $I=0$ ) for a short time, but the infection is reintroduced at a later time. More specifically, the theoretical long-term distribution of the number of infective individuals agrees with the stationary distribution provided in Stone et al. (2007), Section 2.6. 


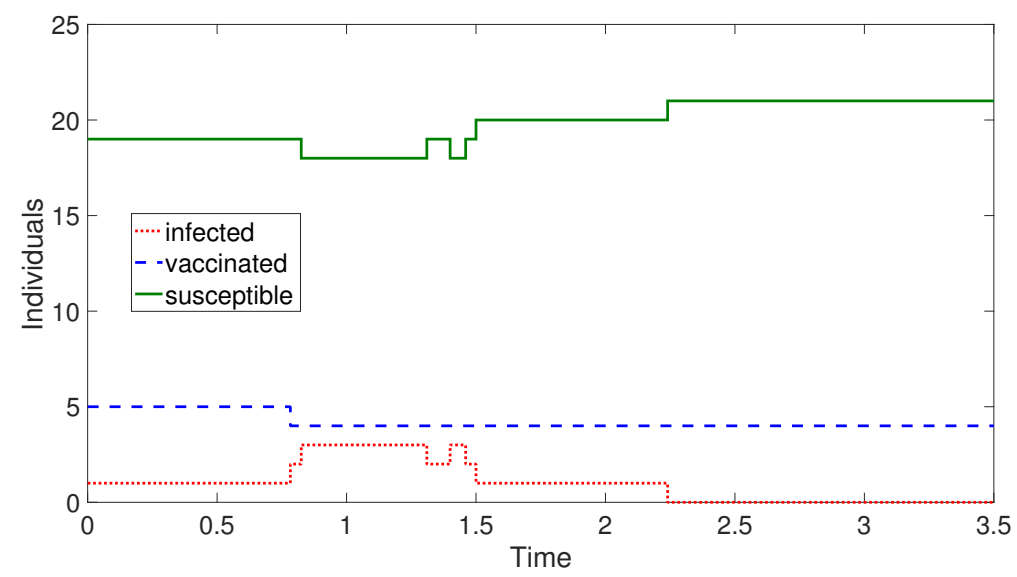

Fig. 3 Simulated trajectories of $X$ when $N=25, \beta=2.5, \xi=0, h=0.3$ and $\gamma=1.0$

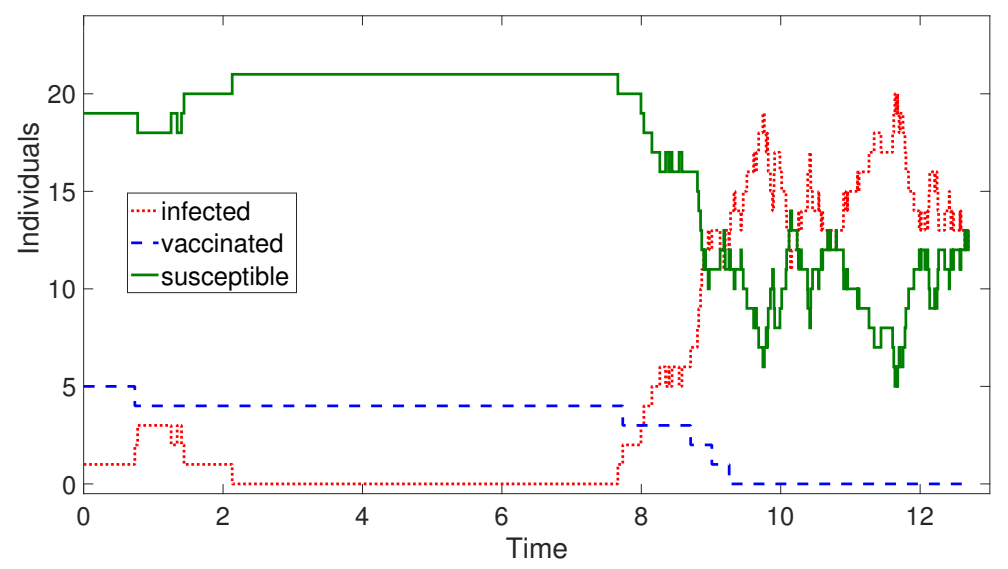

Fig. 4 Simulated trajectories of $X$ when $N=25, \beta=2.5, \xi=0.01, h=0.3$ and $\gamma=1.0$

Figures 3 and 4 show typical trajectories for the CTMC $X$, starting from a single individual infected in a population of 25 individuals, 5 of which are vaccinated. We simulate 500 transitions among states and keep track of the time at which a transition occurs. Each line in Figures 3 and 4 represents the sequence of time points and the number of individuals (infected, vaccinated or susceptible) recorded at these time epochs. The paths in Figure 3 correspond to an isolated population and we observe that, approximately, after 2 time units the epidemic transmission will cease. In Figure 4, the reintroduction parameter 
is $\xi=0.01$. The path for vaccinated individuals shows that vaccine protection has faded away in about 10 time units, while the paths for the infected and susceptible populations settle towards the stationary endemic scenario.

\section{Disease spread}

This section deals with a probabilistic characterization of the offspring distribution of secondary infections of an epidemic process, at a particular time. We will consider two random variables to measure the spread of the infection, when a first case of disease is identified in a population.

In epidemiology, the individual who first brings the disease into a group is called the index case and we will start focusing on the number of infections coming from the index case during its entire infectious period. Secondly, we will study all of the secondary cases produced by the whole set of currently infectious individuals prior to the first recovery. The above mentioned measures are the stochastic analogues to the well-known basic reproduction number $R_{0}$ and, more specifically, to the control reproduction number $R_{c}$, defined as the average number of secondary cases due to each infective individual in the presence of control measures. In the case of vaccination $R_{0}$ and $R_{c}$ satisfy the simple expression $R_{c}=R_{0}(1-(1-h) f)$, where $h$ is the proportion of vaccinated individuals for which the vaccine has no effect (hence, $1-h$ quantifies the vaccine effectiveness), and $f$ represents the vaccine coverage as the initial fraction of the target population that has received the vaccine (Alexander et al. 2004; Magpantay 2014).

The Markovian chain, describing the evolution of the epidemic in the compartmental model, will play an essential role to develop theoretical results. The evaluation of the probability mass distribution of the number of secondary infections produced by a selected individual was firstly introduced by Ross (2011) and, in an independent way, by Artalejo and Lopez-Herrero (2013). Both papers consider finite SIS and SIR compartmental models and present probabilities of the offspring distribution as the solution of systems of linear equations. Their results generalize the study of the infection transmission from the instant of the invasion to any stage of the disease progression and show that, for populations of moderate size, the basic reproduction number can overestimate the potential transmission of the epidemic process.

The analysis of the transmission of an epidemic process has been extended to epidemic models with vector-borne infections (Artalejo 2014), heterogeneous contacts (Economou et al. 2015; López-García 2016), infective and susceptible immigrants (Almaraz et al. 2016), latency periods (LopezHerrero 2017), generally distributed infectious periods (Gómez-Corral and López-García 2017), Markov-modulated interactions (Almaraz and GómezCorral 2018) or to models for two-species competition (Gómez-Corral and López-García 2015).

Our results will reveal the influence of the vaccine, coverage and effectiveness, and the reintroduction parameter on the transmission potential of the 
disease. Hence, in the subsequent sections we proceed with theoretical discussions leading to probabilistic results.

3.1 The exact transmission variable $R_{e 0}$

In this section we study the potential transmission of an infective process by studying the random variable $R_{e 0}$, defined as the number of infective individuals that arise from contagions caused directly by the index case, that is, the first individual in the population able to spread the disease. The objective is to characterize the distribution of the random variable $R_{e 0}$ and to observe the influence of the initial group of vaccinated individuals (i.e., vaccine coverage) on the transmission.

This analysis is directly related to the infectious period of the index case. During this period, the underlying Markov chain $X$ evolves in $\widehat{S}$, the subset of states showing at least one infectious individual. For practical purposes $\widehat{S}$ will be partitioned in levels, according to the current number of vaccinated individuals, as follows:

$$
\widehat{S}=\bigcup_{v=0}^{v_{0}} \widehat{L}(v)=\bigcup_{v=0}^{v_{0}}\{(v, i): 1 \leq i \leq N-v\} .
$$

First, we introduce appropriate notations for the generating and probability mass functions, and for the factorial moments of $R_{e 0}$ conditioned to a specific state $(v, i) \in \widehat{S}$.

$$
\begin{aligned}
\varphi_{v, i}(z) & =E\left[z^{R_{e 0}} \mid(V(0)=v, I(0)=i)\right] \\
& =\sum_{k=0}^{\infty} z^{k} P\left(\left\{R_{e 0}=k \mid(V(0)=v, I(0)=i)\right\}\right), \text { for }|z| \leq 1, \\
x_{v, i}^{k} & =P\left(\left\{R_{e 0}=k \mid(V(0)=v, I(0)=i)\right\}\right), \text { for } k \geq 0, \\
m_{v, i}^{k} & = \begin{cases}1 & \text { if } k=0, \\
E\left[R_{e 0}\left(R_{e 0}-1\right) \cdots\left(R_{e 0}-k+1\right) \mid(V(0)=v, I(0)=i)\right] & \text { if } k>0 .\end{cases}
\end{aligned}
$$

In the rest of the section we will develop algorithmic schemes for determining $\varphi_{v, i}(z), x_{v, i}^{k}$ and the moments $m_{v, i}^{k}$. Firstly, we mark the index case. The homogeneous mixing assumption guarantees all-to-all interactions with no preferences in relationship among individuals. Therefore, individuals are all the same and we split transitions in Table 2, associated to contagions, by distinguishing whether or not a new infection comes from the index case. That is, we partition contagion rates in the following way

$$
\begin{aligned}
\left(\frac{\beta i}{N}+\xi\right)(N-v-i) & =\frac{\beta}{N}(N-v-i)+\left(\frac{\beta(i-1)}{N}+\xi\right)(N-v-i), \\
h\left(\frac{\beta i}{N}+\xi\right) v & =h \frac{\beta}{N} v+h\left(\frac{\beta(i-1)}{N}+\xi\right) v .
\end{aligned}
$$


Then, for $(v, i) \in \widehat{S}$, we define new rates $\alpha_{v, i}^{*}, \widetilde{\alpha}_{v, i}, \beta_{v}^{*}$ and $\widetilde{\beta}_{v, i}$ as follows.

$$
\begin{aligned}
\alpha_{v, i}^{*} & =\frac{\beta}{N}(N-v-i), \\
\widetilde{\alpha}_{v, i} & =\left(\frac{\beta(i-1)}{N}+\xi\right)(N-v-i), \\
\beta_{v}^{*} & =h \frac{\beta}{N} v, \\
\widetilde{\beta}_{v, i} & =h\left(\frac{\beta(i-1)}{N}+\xi\right) v .
\end{aligned}
$$

A first-step argument, conditioning on the possible transitions out of a fixed state $(v, i) \in \widehat{S}$, shows that generating functions $\varphi_{v, i}(z)$ satisfy the following set of linear equations:

$$
\begin{aligned}
\varphi_{v, i}(z)= & \frac{\gamma}{q_{v, i}}+\left(1-\delta_{v, 0}\right)\left(\frac{\beta_{v}^{*}}{q_{v, i}} z \varphi_{v-1, i+1}(z)+\frac{\widetilde{\beta}_{v, i}}{q_{v, i}} \varphi_{v-1, i+1}(z)\right) \\
& +\left(1-\delta_{i, 1}\right) \frac{\gamma(i-1)}{q_{v, i}} \varphi_{v, i-1}(z) \\
& +\left(1-\delta_{i, N-v}\right)\left(\frac{\alpha_{v, i}^{*}}{q_{v, i}} z \varphi_{v, i+1}(z)+\frac{\widetilde{\alpha}_{v, i}}{q_{v, i}} \varphi_{v, i+1}(z)\right),
\end{aligned}
$$

where $\delta_{i, j}$ represents the Kronecker's delta function, defined as 1 when $i=j$, and 0 otherwise.

Equation (6) is the basis to get the mass function of the conditional random variable $R_{e 0} \mid(V(0)=v, I(0)=i)$ by numerical inversion, which can be done with the help of a Fast Fourier Transform (FFT) algorithm (Tijms 2003). As mass functions will be obtained through a direct recursive scheme, we do not go further on this point and we proceed to get factorial moments $m_{v, i}^{k}$, for $k \geq 1$.

By differentiating equation (6) with respect to $z$ repeatedly $k$ times $(k \geq 1)$ and evaluating at $z=1$, we obtain the equations involving factorial moments conditioned to states $(v, i) \in \widehat{S}$.

$$
\begin{aligned}
m_{v, i}^{k}= & \left(1-\delta_{v, 0}\right)\left(\frac{\beta_{v}^{*}}{q_{v, i}} m_{v-1, i+1}^{k}+\frac{\widetilde{\beta}_{v, i}}{q_{v, i}} m_{v-1, i+1}^{k}\right)+\left(1-\delta_{i, 1}\right) \frac{\gamma(i-1)}{q_{v, i}} m_{v, i-1}^{k} \\
& +\left(1-\delta_{i, N-v}\right)\left(\frac{\alpha_{v, i}^{*}}{q_{v, i}} m_{v, i+1}^{k}+\frac{\widetilde{\alpha}_{v, i}}{q_{v, i}} m_{v, i+1}^{k}\right) \\
& +\left(1-\delta_{v, 0}\right) k \frac{\beta_{v}^{*}}{q_{v, i}} m_{v-1, i+1}^{k-1}+\left(1-\delta_{i, N-v}\right) k \frac{\alpha_{v, i}^{*}}{q_{v, i}} m_{v, i+1}^{k-1} .
\end{aligned}
$$

Using relationships appearing in (2)-(5), we can write a useful and simplified version of (7),

$$
q_{v, i} m_{v, i}^{k}=\left(1-\delta_{v, 0}\right) h\left(\frac{\beta i}{N}+\xi\right) v m_{v-1, i+1}^{k}+\left(1-\delta_{i, 1}\right) \gamma(i-1) m_{v, i-1}^{k}
$$




$$
\begin{aligned}
& +\left(1-\delta_{i, N-v}\right)\left(\frac{\beta i}{N}+\xi\right)(N-v-i) m_{v, i+1}^{k} \\
& +\left(1-\delta_{v, 0}\right) k \beta_{v}^{*} m_{v-1, i+1}^{k-1}+\left(1-\delta_{i, N-v}\right) k \alpha_{v, i}^{*} m_{v, i+1}^{k-1}
\end{aligned}
$$

We notice that equation (8) provides conditional moments of order $k$ based on conditional moments of one order less.

Moreover, at any level $0 \leq v \leq v_{0}$, the system of equations described in (8), for $1 \leq i \leq N-v$ and $k \geq 0$, can be written in matrix form as follows

$$
\begin{aligned}
\mathbf{m}_{\mathbf{v}}^{\mathbf{0}} & =\mathbf{e}_{\mathbf{v}}, \\
\mathbf{A}_{\mathbf{v}} \mathbf{m}_{\mathbf{v}}^{\mathbf{k}} & =-\left(\left(1-\delta_{v, 0}\right)\left(\mathbf{D}_{\mathbf{v}} \widehat{\mathbf{m}}_{\mathbf{v}-\mathbf{1}}^{\mathbf{k}}+\mathbf{D}_{\beta \mathbf{v}} \widehat{\mathbf{m}}_{\mathbf{v}-\mathbf{1}}^{\mathbf{k}-\mathbf{1}}\right)+k \mathbf{D}_{\alpha \mathbf{v}} \widetilde{\mathbf{m}}_{\mathbf{v}}^{\mathbf{k}-\mathbf{1}}\right),
\end{aligned}
$$

where the auxiliary matrices and vectors involved in (9)-(10) are defined below.

For $0 \leq v \leq v_{0}, \mathbf{e}_{\mathbf{v}}$ is an all-ones vector of dimension $(N-v)$ and $\mathbf{A}_{\mathbf{v}}$ will denote the square $(N-v)$ matrix with non null entries

$$
A_{v}(i, j)= \begin{cases}(i-1) \gamma & \text { if } j=i-1,2 \leq i \leq N-v \\ -q_{v, i} & \text { if } j=i, 1 \leq i \leq N-v \\ \left(\frac{\beta i}{N}+\xi\right)(N-v-i) & \text { if } j=i+1,1 \leq i \leq N-v-1 .\end{cases}
$$

In addition, $\mathbf{D}_{\mathbf{v}}, \mathbf{D}_{\beta \mathbf{v}}$ and $\mathbf{D}_{\alpha \mathbf{v}}$ represent $(N-v)$ diagonal matrices, defined as follows:

$$
\begin{aligned}
\mathbf{D}_{\mathbf{v}} & =\operatorname{Diag}\left(h\left(\frac{\beta i}{N}+\xi\right) v: 1 \leq i \leq N-v\right) \\
\mathbf{D}_{\beta \mathbf{v}} & =\operatorname{Diag}\left(\beta_{v}^{*}: 1 \leq i \leq N-v\right) \\
\mathbf{D}_{\alpha \mathbf{v}} & =\operatorname{Diag}\left(\alpha_{v, i}^{*}: 1 \leq i \leq N-v\right)
\end{aligned}
$$

Finally, for $k \geq 0$ and $0 \leq v \leq v_{0}, \mathbf{m}_{\mathbf{v}}^{\mathbf{k}}$ and $\widetilde{\mathbf{m}}_{\mathbf{v}}^{\mathbf{k}}$ are $(N-v)$-dimensional vectors defined as

$$
\begin{aligned}
& \mathbf{m}_{\mathbf{v}}^{\mathbf{k}}=\left(m_{v, 1}^{k}, \ldots, m_{v, N-v}^{k}\right)^{T}, \\
& \widetilde{\mathbf{m}}_{\mathbf{v}}^{\mathbf{k}}=\left(m_{v, 2}^{k}, \ldots, m_{v, N-v}^{k}, 0\right)^{T},
\end{aligned}
$$

and $\widehat{\mathbf{m}}_{\mathbf{v}}^{\mathbf{k}}$ is an $(N-v-1)$-dimensional vector given by $\widehat{\mathbf{m}}_{\mathbf{v}}^{\mathbf{k}}=\left(m_{v, 2}^{k}, \ldots, m_{v, N-v}^{k}\right)^{T}$, where notation $T$ denotes transposition.

Given an integer $k$, factorial moments are recursively determined with the help of the algorithmic scheme shown in Algorithm 1.

Remark 1 Notice that the random variable $R_{e 0}$ measures the exact number of secondary infective individuals arising directly from the index case and, in contradistinction with $R_{0}$ or $R_{c}$ that are defined at the time of the invasion, $R_{e 0}$ can be checked at all times. If we set $t=0$ for the time at which the invasion starts the initial situation is $V(0)=v_{0}$ and $I(0)=1$, and $\bar{R}_{e 0}=E\left[R_{e 0} \mid V(0)=v_{0}, I(0)=1\right]$ provides the exact amount of expected disease transmission, considering vaccine characteristics such as coverage and effectiveness. 


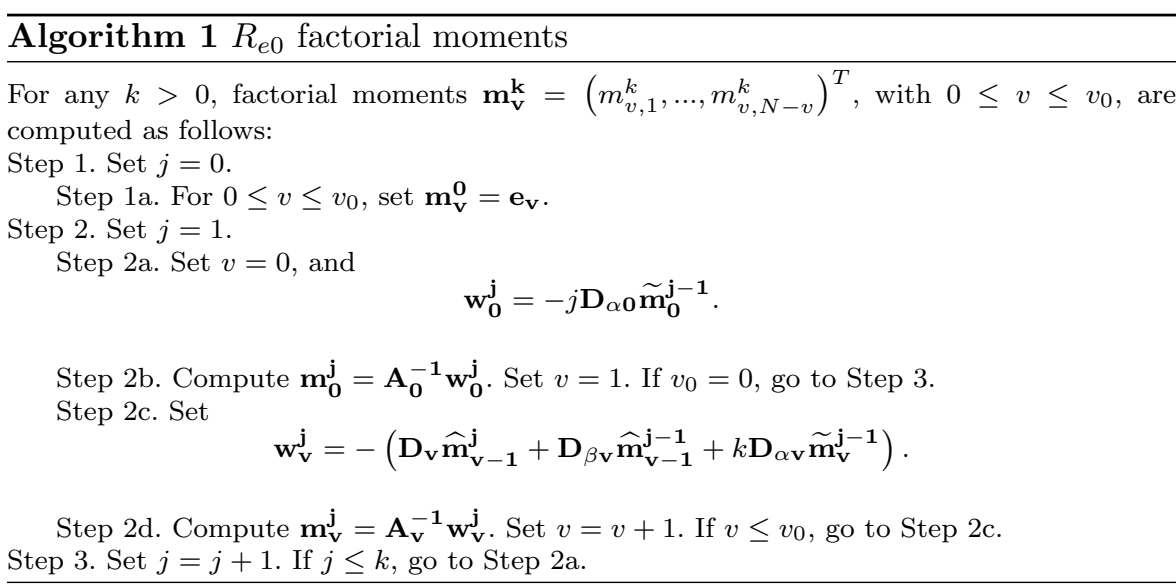

Let us proceed to the analytic derivation of the probabilities $x_{v, i}^{k}$ that the index case will originate $k \geq 0$ new infections, given that the current situation is $(v, i) \in \widehat{S}$. A new appeal to the first-step methodology, by observing transitions out of the state $(v, i)$, provides the following set of recursive equations:

$$
\begin{aligned}
q_{v, i} x_{v, i}^{k}= & \delta_{k, 0} \gamma+\left(1-\delta_{k, 0}\right)\left(\left(1-\delta_{v, 0}\right) \beta_{v}^{*} x_{v-1, i+1}^{k-1}+\left(1-\delta_{i, N-v}\right) \alpha_{v, i}^{*} x_{v, i+1}^{k-1}\right) \\
& +\left(1-\delta_{v, 0}\right) \widetilde{\beta}_{v, i} x_{v-1, i+1}^{k} \\
& +(i-1) \gamma x_{v, i-1}^{k}+\left(1-\delta_{i, N-v}\right) \widetilde{\alpha}_{v, i} x_{v, i+1}^{k} .
\end{aligned}
$$

For $k \geq 0$ and $0 \leq v \leq v_{0}$, equation (11) for states in $L(v)$ can be written in matrix form in the following way:

$$
\begin{aligned}
& \mathbf{B}_{\mathbf{v}} \mathbf{x}_{\mathbf{v}}^{\mathbf{0}}=-\gamma \mathbf{e}_{\mathbf{v}}-\left(1-\delta_{v, 0}\right) \mathbf{C}_{\mathbf{v}} \widehat{\mathbf{x}}_{\mathbf{v}-\mathbf{1}}^{\mathbf{0}}, \\
& \mathbf{B}_{\mathbf{v}} \mathbf{x}_{\mathbf{v}}^{\mathbf{k}}=-\left(1-\delta_{v, 0}\right)\left(\mathbf{C}_{\mathbf{v}} \widehat{\mathbf{x}}_{\mathbf{v}-\mathbf{1}}^{\mathbf{k}}+\mathbf{D}_{\beta \mathbf{v}} \widehat{\mathbf{x}}_{\mathbf{v}-\mathbf{1}}^{\mathbf{k}-\mathbf{1}}\right)-\mathbf{D}_{\alpha \mathbf{v}} \widetilde{\mathbf{x}}_{\mathbf{v}}^{\mathbf{k}-\mathbf{1}}, k>0,
\end{aligned}
$$

where notations $\mathbf{e}_{\mathbf{v}}, \mathbf{D}_{\beta \mathbf{v}}$ and $\mathbf{D}_{\alpha \mathbf{v}}$ represent the same algebraic structures as defined in the lines following equations (9)-(10).

The remaining algebraic structures appearing in matrix expressions (12) are described next: $\mathbf{B}_{\mathbf{v}}$ and $\mathbf{C}_{\mathbf{v}}$ are $(N-v)$ square matrices with entries

$$
\begin{aligned}
B_{v}(i, j) & = \begin{cases}(i-1) \gamma & \text { if } j=i-1,2 \leq i \leq N-v, \\
-q_{v, i} & \text { if } j=i, 1 \leq i \leq N-v, \\
\widetilde{\alpha}_{v, i} & \text { if } j=i+1,1 \leq i \leq N-v-1, \\
0 & \text { otherwise }\end{cases} \\
\mathbf{C}_{\mathbf{v}} & =\operatorname{Diag}\left(\widetilde{\beta}_{v, i}: 1 \leq i \leq N-v\right),
\end{aligned}
$$

and the vectors $\mathbf{x}_{\mathbf{v}}^{\mathbf{k}}, \widehat{\mathbf{x}}_{\mathbf{v}}^{\mathbf{k}}$ and $\widetilde{\mathbf{x}}_{\mathbf{v}}^{\mathbf{k}}$, containing probabilities $x_{v, i}^{k}$, are

$$
\mathbf{x}_{\mathbf{v}}^{\mathbf{k}}=\left(x_{v, 1}^{k}, \ldots, x_{v, N-v}^{k}\right)^{T},
$$




$$
\begin{aligned}
& \widehat{\mathbf{x}}_{\mathbf{v}}^{\mathbf{k}}=\left(x_{v, 2}^{k}, \ldots, x_{v, N-v}^{k}\right)^{T} \\
& \widetilde{\mathbf{x}}_{\mathbf{v}}^{\mathbf{k}}=\left(x_{v, 2}^{k}, \ldots, x_{v, N-v}^{k}, 0\right)^{T} .
\end{aligned}
$$

Notice that $P\left(\left\{R_{e 0}<\infty \mid V(0)=v, I(0)=i\right\}\right)=1$, for $(v, i) \in \widehat{S}$, because $\widehat{S}$ is a finite union of disjoint finite sets, amd in a finite population the number of infective individuals arising from contagions caused by the index case is necessarily finite. Consequently, $\sum_{k=0}^{\infty} x_{v, i}^{k}=1$, for $(v, i) \in \widehat{S}$.

For every number of contagions $k \geq 0$ the equations in (12) are solved recursively with the help of Algorithm 2. In order to determine mass distribution functions, a stopping criteria should be provided to avoid longer computation runs. In fact, numerical results appearing in Section 4 come from applying both recursive Algorithms 1 and 2. More specifically, those results dealing with $R_{e 0}$-distributions for a given initial coverage value, $v_{0}$, are obtained from iterating Algorithm 2 until $95 \%$ of the values of the distribution of $\left(R_{e 0} \mid V(0)=v_{0}, I(0)=1\right)$ is accumulated.

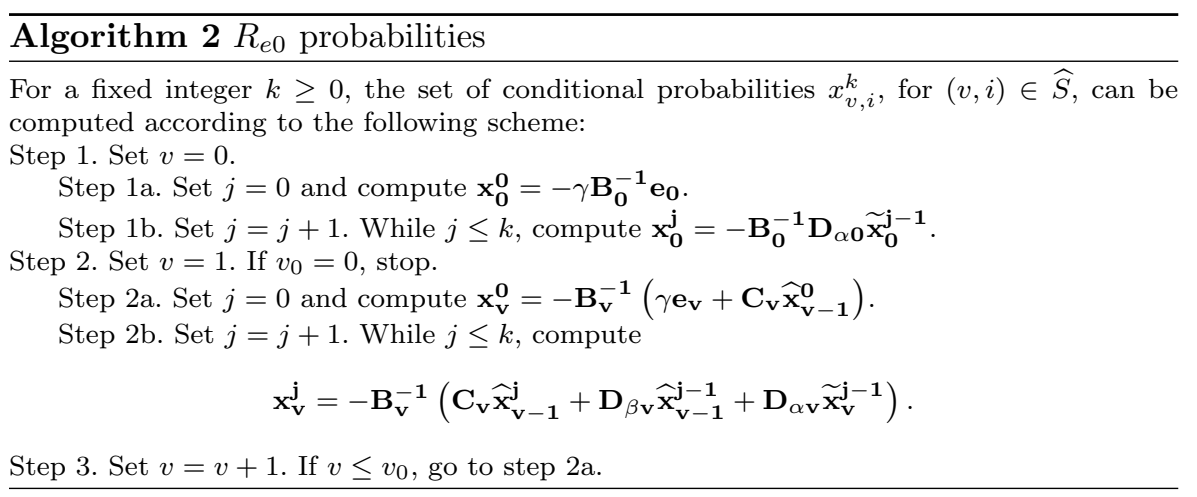

\subsection{The population transmission variable}

Another measure of the expansion of a contagious disease is $R_{p}$, which provides the global spread of the disease by counting all the infections that take place within the population, no matter who is the infectious spreader, before the first recovery occurs. $R_{p}$ is a random variable that can be analyzed either at the beginning of the outbreak or at any later time, by updating the population situation in terms of the current state of the CTMC $X$.

The aim of this section is to describe the probabilistic behavior of $R_{p}$ and to study the influence of the model parameters on global infection transmission. In particular, we are interested in comparing $R_{c}$, the control reproduction number of this model, with the expected value $\bar{R}_{p}=E\left[R_{p} \mid\left(V(0)=v_{0}, I(0)=\right.\right.$ 1)] for different scenarios.

First, we derive theoretical results involving the probability distribution and factorial moments of $R_{p}$. As in the preceding section, the central tool for 
our results will be the first-step methodology. But to avoid repetitive arguments we provide results in a comprehensive manner, leaving out unnecessary details.

Let $\psi_{v, i}(z)$ be the generating function of $R_{p}$, given that the current state of $X$ is $(v, i) \in \widehat{S}$, with factorial moments $M_{v, i}^{k}$ for $k \geq 1\left(M_{v, i}^{0}=1\right)$. At any point $z$, with $|z| \leq 1$, generating functions are the solution of the following tridiagonal set of linear equations:

$$
\begin{aligned}
\psi_{v, i}(z)= & \frac{\gamma i}{q_{v, i}}+\left(1-\delta_{v, 0}\right) \frac{h\left(\frac{\beta i}{N}+\xi\right) v}{q_{v, i}} z \psi_{v-1, i+1}(z) \\
& +\left(1-\delta_{i, N-v}\right) \frac{\left(\frac{\beta i}{N}+\xi\right)(N-v-i)}{q_{v, i}} z \psi_{v, i+1}(z),
\end{aligned}
$$

for $0 \leq v \leq v_{0}, 1 \leq i \leq N-v$.

The $k$-th factorial moments of states $(v, i) \in \widehat{S}$ are the solution of a system of linear equations, arising from (13) as usual by taking derivatives with respect to $z$ followed by an evaluation for $z=1$, that is expressed in matrix form as follows:

$$
\mathbf{H}_{\mathbf{v}} \mathbf{M}_{\mathbf{v}}^{\mathbf{k}}=-\left(1-\delta_{v, 0}\right) \mathbf{D}_{\mathbf{v}}\left(k \widehat{\mathbf{M}}_{\mathbf{v}-\mathbf{1}}^{\mathbf{k}-\mathbf{1}}+\widehat{\mathbf{M}}_{\mathbf{v}-1}^{\mathbf{k}}\right)-k \widetilde{\mathbf{D}}_{\mathbf{v}} \widetilde{\mathbf{M}}_{\mathbf{v}}^{\mathbf{k}-\mathbf{1}}
$$

for $k \geq 1$ and $0 \leq v \leq v_{0}$. Here $\mathbf{H}_{\mathbf{v}}$ and $\widetilde{\mathbf{D}}_{\mathbf{v}}$ are $(N-v)$ square matrices defined by:

$$
\begin{gathered}
H_{v}(i, j)= \begin{cases}-q_{v, i} & \text { if } j=i, 1 \leq i \leq N-v \\
\left(\frac{\beta i}{N}+\xi\right)(N-v-i) & \text { if } j=i+1,1 \leq i \leq N-v-1, \\
0 & \text { otherwise. }\end{cases} \\
\widetilde{\mathbf{D}}_{\mathbf{v}}=\operatorname{Diag}\left(\left(\frac{\beta i}{N}+\xi\right)(N-v-i): 1 \leq i \leq N-v\right)
\end{gathered}
$$

Vectors $\mathbf{M}_{\mathbf{v}}^{\mathbf{k}}, \widehat{\mathbf{M}}_{\mathbf{v}}^{\mathbf{k}}$ and $\widetilde{\mathbf{M}}_{\mathbf{v}}^{\mathbf{k}}$ related to factorial moments are defined as follows

$$
\begin{aligned}
& \mathbf{M}_{\mathbf{v}}^{\mathbf{k}}=\left(M_{v, 1}^{k}, \ldots, M_{v, N-v}^{k}\right)^{T}, \\
& \widehat{\mathbf{M}}_{\mathbf{v}}^{\mathbf{k}}=\left(M_{v, 2}^{k}, \ldots, M_{v, N-v}^{k}\right)^{T} \\
& \widetilde{\mathbf{M}}_{\mathbf{v}}^{\mathbf{k}}=\left(M_{v, 2}^{k}, \ldots, M_{v, N-v}^{k}, 0\right)^{T} .
\end{aligned}
$$

The distribution of the random variable $R_{p}$, conditioned to any state $(v, i) \in \widehat{S}$, can be obtained by inverting transforms with the help of the recursive equations (13) and an FFT algorithm. But, as it was stated in the preceding section, it is possible to find a set of equations whose solution provides directly the point mass function of $R_{p}: z_{v, i}^{k}=P\left(\left\{R_{p}=k \mid V(0)=v, I(0)=i\right\}\right)$, 
when $(v, i) \in \widehat{S}$, for $0 \leq k \leq N-1$. Notice that when we observe $i$ infected individuals in the population, then the number of secondary cases taking place before the first recovery is at most $N-i$. So, for any integer $k$, with $0 \leq k \leq N-1$, $z_{v, i}^{k}=0$, whenever $i>N-k$.

Next, we introduce appropriate vectors to derive probabilities $z_{v, i}^{k}$ when $(v, i) \in \widehat{S}$, that is:

$$
\begin{aligned}
& \mathbf{z}_{\mathbf{v}}^{\mathbf{k}}=\left(z_{v, 1}^{k}, \ldots, z_{v, N-v}^{k}\right)^{T}, \\
& \widehat{\mathbf{z}}_{\mathbf{v}}^{\mathbf{k}}=\left(z_{v, 2}^{k}, \ldots, z_{v, N-v}^{k}\right)^{T}, \\
& \widetilde{\mathbf{z}}_{\mathbf{v}}^{\mathbf{k}}=\left(z_{v, 2}^{k}, \ldots, z_{v, N-v}^{k}, 0\right)^{T} .
\end{aligned}
$$

Finally, for each level $v$, with $0 \leq v \leq v_{0}, R_{p}$ point probabilities come from the following recursive equations:

$$
\begin{aligned}
& \mathbf{D}_{\mathbf{Q}} \mathbf{z}_{\mathbf{v}}^{\mathbf{0}}=\mathbf{d}_{\gamma}, \\
& \mathbf{D}_{\mathbf{Q}} \mathbf{z}_{\mathbf{v}}^{\mathbf{k}}=\left(1-\delta_{v, 0}\right) \mathbf{D}_{\mathbf{v}} \widehat{\mathbf{z}}_{\mathbf{v}-1}^{\mathbf{k}-\mathbf{1}}+\widetilde{\mathbf{D}}_{\mathbf{v}} \widetilde{\mathbf{z}}_{\mathbf{v}}^{\mathbf{k}-\mathbf{1}}, \text { for } 1 \leq k \leq N-1,
\end{aligned}
$$

where $\mathbf{D}_{\mathbf{Q}}$ and $\mathbf{d}_{\gamma}$ are a diagonal matrix and a vector, with respective entries $q_{v, i}$ and $\gamma i$, for $1 \leq i \leq N-v$.

As in Section 3.1, $R_{p}$ factorial moments and probabilities can be computed from recursive schemes based on equations (14) and (15 - 16), respectively. Their algorithmic descriptions are similar to Algorithms $1-2$ and are not stated in the text, but they are the basis for obtaining $R_{p}$ 's numerical results appearing in Section 4.

Remark 2 In the paper by Artalejo and Lopez-Herrero (2014), the authors presented closed form expressions for probabilities dealing with the population transmission random variable in stochastic SIS and SIR models. For our SIV model, the mathematics is more involved due to the external transmission parameter and, unfortunately, it is not possible to derive general closed expressions for conditional probabilities $z_{v, i}^{k}$. However, after some algebra we obtained the closed values of point probabilities in a few specific situations.

$$
z_{0, N}^{k}=P\left(\left\{R_{p}=k \mid V(0)=0, I(0)=N\right\}\right)=\delta_{k, 0}, \text { for } 0 \leq k \leq N-1 .
$$

For $0 \leq v \leq v_{0}$ and $1 \leq i \leq N-v$ we get:

$$
\begin{aligned}
z_{v, i}^{0} & =P\left(\left\{R_{p}=0 \mid V(0)=v, I(0)=i\right\}\right)=\frac{\gamma i}{q_{v, i}}, \\
z_{v, i}^{1} & =P\left(\left\{R_{p}=1 \mid V(0)=v, I(0)=i\right\}\right) \\
& =\left(1-\delta_{i, N}\right) \frac{\left(\frac{\beta}{N} i+\xi\right) \gamma(i+1)}{q_{v, i}}\left(\frac{h v}{q_{v-1, i+1}}+\frac{(N-v-i)}{q_{v, i+1}}\right) .
\end{aligned}
$$




\section{Numerical illustrations}

This section illustrates theoretical and algorithmic results derived in previous sections.

We fix the recovery rate as $\gamma=1.0$ in all the experiments, so the time unit is taken as to be the expected time that an infected individual takes to recover to become susceptible again.

In the first scenario, we consider a population of $N=100$ individuals, $20 \%$ of which is partially protected against the infection by a vaccine with effectiveness of $97 \%$. We are interested in the random variable $R_{e 0}$, that is the number of secondary infections produced by the index case. Hence, the initial number of infective individuals is $I(0)=i_{0}=1$.

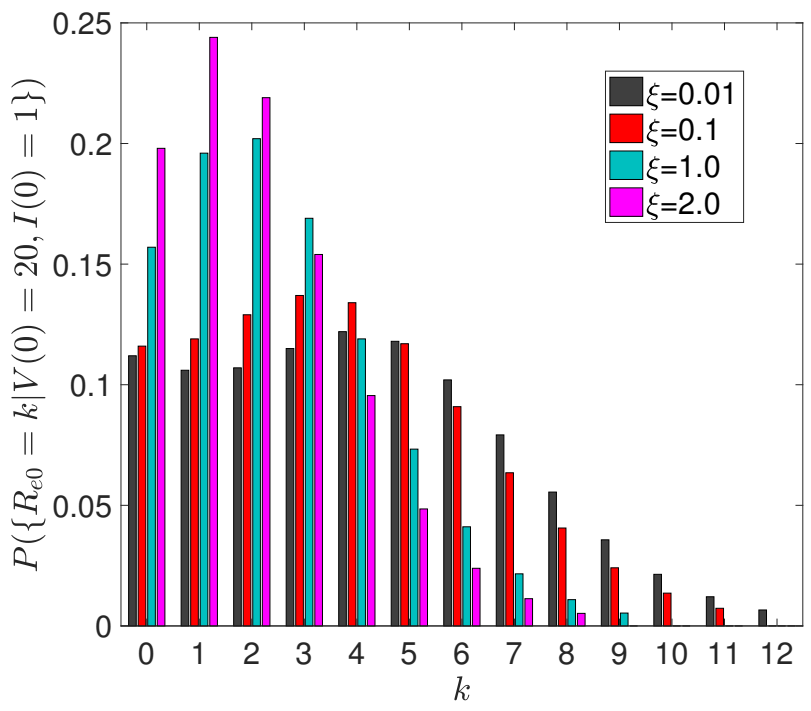

Fig. $5 R_{e 0}$ distribution for several values of $\xi$, when $N=100, \beta=10.0$ and $h=0.03$

Figures 5 and 6 represent histograms for the distribution of $R_{e 0}$ when we vary external or internal contact rates, respectively. Heights indicate the value of the probabilities $x_{20,1}^{k}=P\left(\left\{R_{e 0}=k \mid V(0)=20, I(0)=1\right\}\right)$ and colors appearing in both figures are depicted for a better distinction among considered situations. In more detail, Figure 5 shows mass functions of $R_{e 0}$ when the external transmission rate $\xi$ is $0.01,0.1,1.0$ and 2.0, for a fixed internal transmission rate $\beta=10.0$. For each rate $\xi$, mean values of $R_{e 0}$ are $4.2035,3.7601,2.5342$ and 2.0592, respectively. Moreover, distributions present a strictly positive mode that occurs with a probability that decreases for increasing values of the external rate. This remark is according to the intuition because for a fixed internal contact transmission rate $\beta$, when the external transmission rate in- 
creases the index case has less opportunities to spread the disease compared with outsider infection sources.

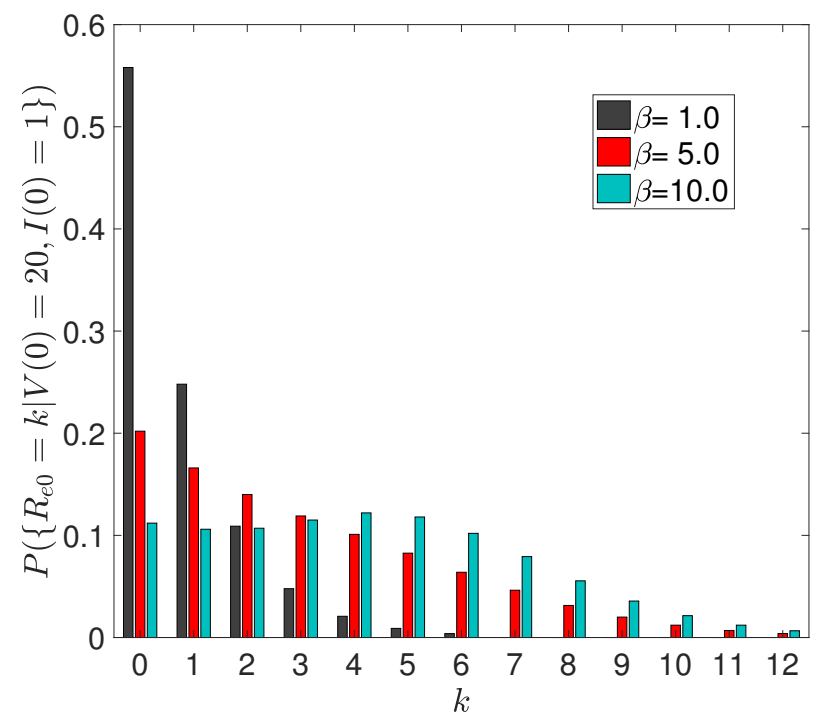

Fig. $6 R_{e 0}$ distribution for several values of $\beta$, when $N=100, \xi=0.01$ and $h=0.03$

Figure 6 displays mass functions corresponding to $\beta=1.0,5.0$ and 10.0, for an external transmission rate $\xi=0.01$. We get decreasing shape functions for $\beta=1.0$ or $\beta=5.0$ and the chance that the index case produces no secondary infections is $55 \%$ and $20 \%$, respectively. However, for $\beta=10.0$, the $R_{e 0}$ distribution is bimodal, with an $11.2 \%$ chance that the index case recovers before spreading the infection. In general terms, long internal transmission rates contribute a higher number of secondary cases.

Next we focus on the effect of the vaccine coverage on the expansion of the infection. Figure 7 shows the probability that the index case produces two or more secondary cases of infection, as a function of the external rate $\beta$. This quantity can give an idea of what is, for a given infective process, the chance of invading a susceptible-vaccinated population. Each curve corresponds to a different initial vaccine coverage. The remaining parameters of the model are $N=100, \xi=0.01$, and the vaccine is efficient in $97 \%$ of the vaccinated individuals. Probability increases with $\beta$, no matter how large the initial coverage $v_{0}$ is. For a fixed external transmission rate, the chance of having at least two infections increases when initial coverage decreases. In particular for a small population of 100 individuals affected by a hard measles outbreak, with $R_{0}=\beta / \gamma=18.0$, numerical results evince that a massive vaccination policy with two doses of MMR vaccine (measles, mumps, rubella) guarantees that the probability of having two or more measles infections from the index case 
is 0.12 , while in an unprotected population $\left(v_{0}=0\right)$ this probability grows up to 0.9 .

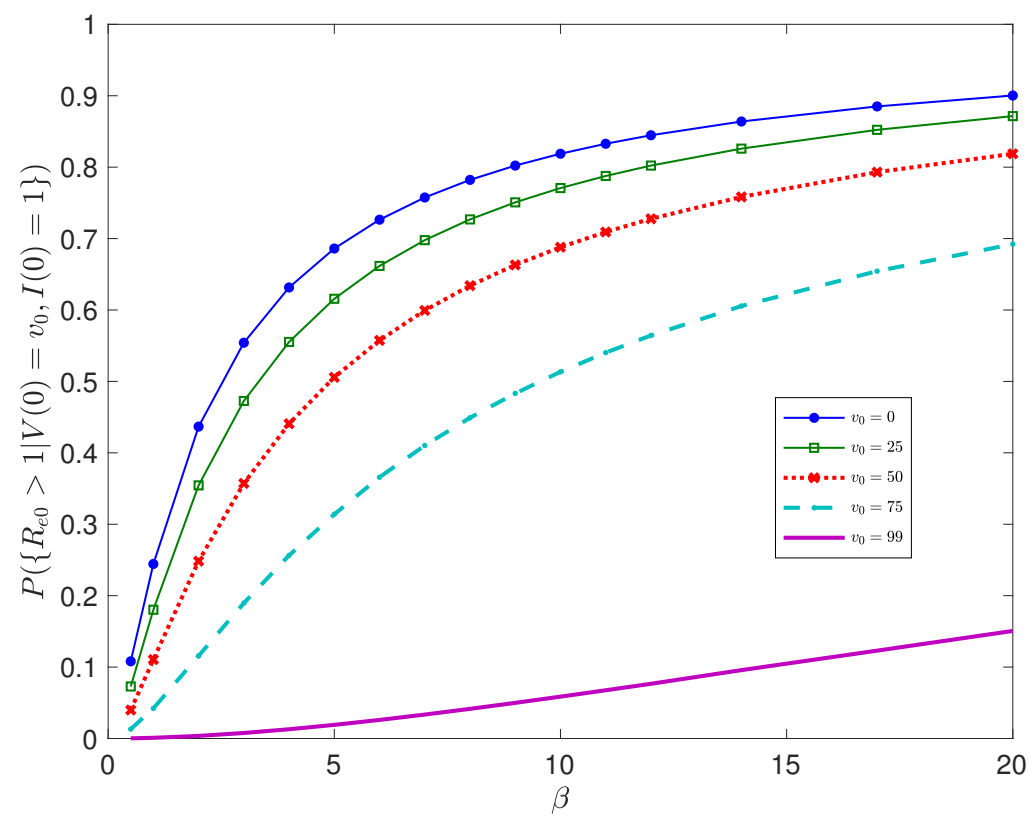

Fig. $7 P\left(\left\{R_{e 0}>1 \mid V(0)=v_{0}, I(0)=1\right\}\right)$ as a function of the internal transmission rate when $N=100, \xi=0.01$ and $h=0.03$

Now we deal with the expected number of secondary cases produced by the index case since their introduction in a population where individuals are either susceptible or vaccinated. We observe the influence of the model parameters $\beta$, $\xi, h$ and $v_{0}$ on the expected value of $R_{e 0}$. Results correspond to a population of $N=100$ individuals.

Figures 8-10 are contour graphs for $\bar{R}_{e 0}=E\left[R_{e 0} \mid V(0)=v_{0}, I(0)=1\right]$, arising when we combine the influence of two parameters of the model. Different colors represent different values for $\bar{R}_{e 0}$, as it is indicated by the color code given in each of the Figures.

Figure 8 shows the influence on $\bar{R}_{e 0}$ of the internal and external transmission rates, when $20 \%$ of the population has received a vaccine which is effective among $97 \%$ of the vaccinated individuals. The average of secondary infections, produced by the index case, increases with the internal transmission rate and it decreases when $\xi$ increases, which is in agreement with the comment for Figure 5 . These behaviors are more noticeable for large transmission intensities.

Figure 9 displays $\bar{R}_{e 0}$ as a function of the internal transmission rate, $\beta$, and the potential risk of vaccine failure, $h$. We assume in addition that $20 \%$ of the 
population is vaccinated and that the rate of external transmission is $\xi=0.01$. The expected number of infections caused directly by the index case increases with $\beta$. In the early spread of the epidemic, the influence of the vaccine failure risk is relatively small for outbreaks showing internal rates $\beta$ smaller than 6 compared to those with higher values for $\beta$.

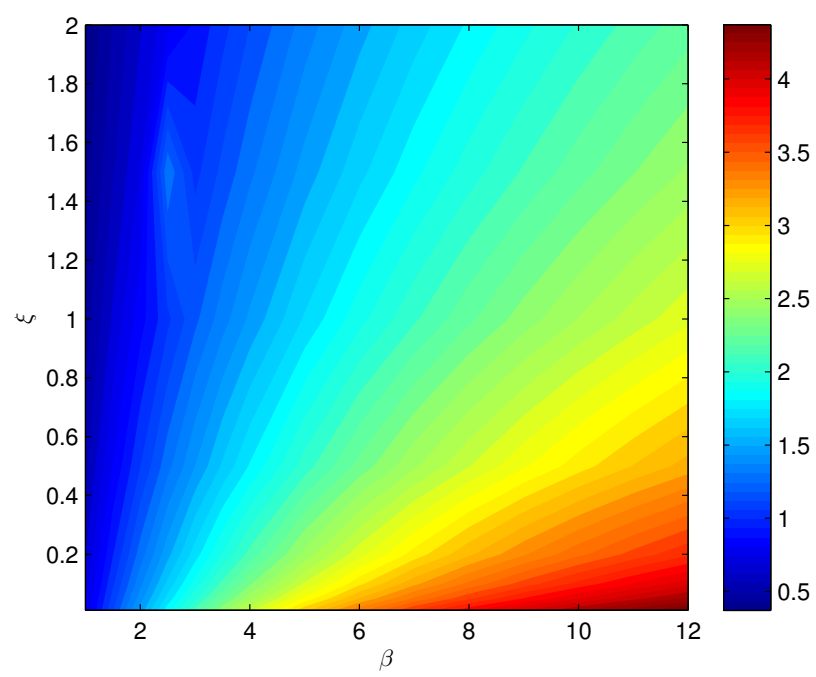

Fig. $8 \bar{R}_{e 0}$ as a function of $\beta$ and $\xi$ when $N=100, h=0.03$ and $v_{0}=20$

Finally, the contour graph shown in Figure 10 presents the relationship of the internal transmission and vaccine coverage on $\bar{R}_{e 0}$. The additional parameters are $N=100, \xi=0.01$ and $97 \%$ for vaccine effectiveness. An increase of vaccine coverage produces a decrease on the initial spread of the infection. For instance, administration of two doses of MMR vaccine is $97 \%$ effective against rubella (basic reproduction number $R_{0} \approx 6$ ). So, assuming that $R_{0}=\beta / \gamma$, results in Figure 10 show that the index case transmits an infection like rubella to an average of 3.5 people in an unprotected population, and to less than one person when a massive vaccination policy was launched in the population. To achieve that the infection will not propagate in the population, that is $R_{e 0}<1$, higher immunization coverage is needed as $\beta$ increases. 


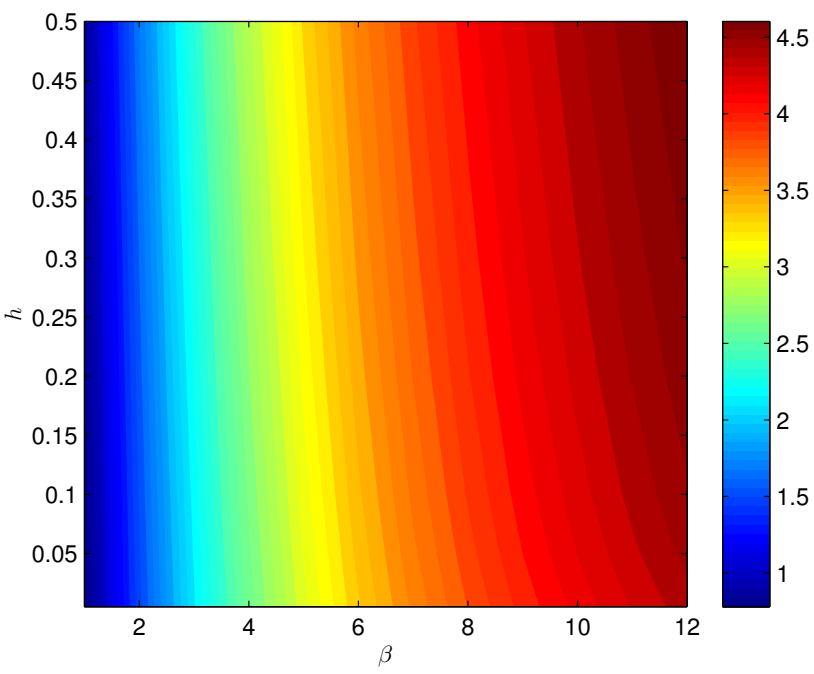

Fig. $9 \bar{R}_{e 0}$ as a function of $\beta$ and $h$ when $N=100, \xi=0.01$ and $v_{0}=20$

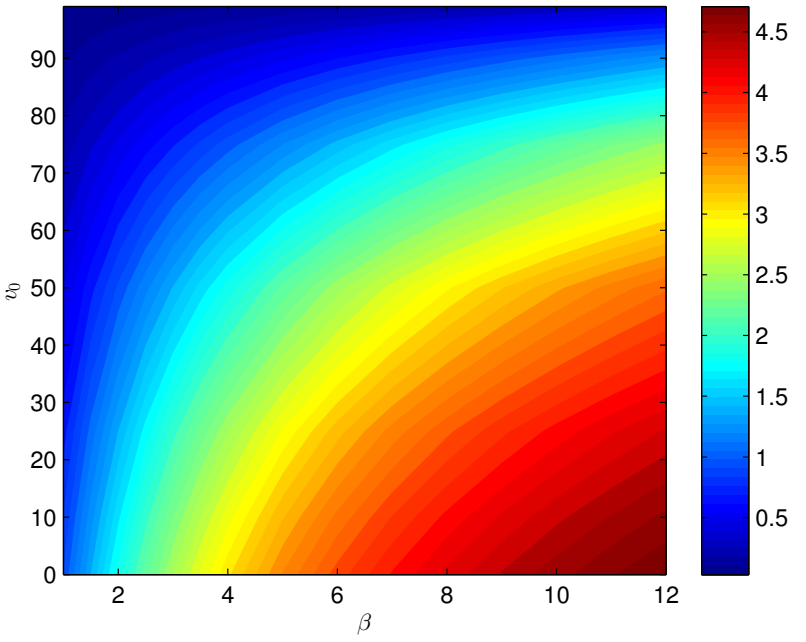

Fig. $10 \bar{R}_{e 0}$ as a function of $\beta$ and $v_{0}$ when $N=100, \xi=0.01$ and $h=0.03$

The boxplot appearing in Figure 11 corresponds to the distribution of the number of secondary infections produced by the index case. The box encloses the middle central part of the distribution, lower and upper edges of the box 
correspond to the lower and upper quartile, respectively, and the line drawn across the box indicates the median of the distribution; finally, whiskers above and below the box cover $95 \%$ of the distribution. The objective is to compare the patterns of the epidemic when we increase the vaccination coverage, in a population of $N=100$ individuals, assuming that a 97\%-effective vaccine is available to control an epidemic process with internal and external transmission rates $\beta=10.0$ and $\xi=0.1$, respectively. This choice for model parameters corresponds to an infection by varicella-zoster virus (VZV) and the administration of varicella vaccine that is $97 \%$ effective in the first year after vaccination. A low vaccine coverage leads to a large number of secondary infections. In general terms, the number of secondary cases produced by the index case decreases when $v_{0}$ increases. Notice that changes on the number of secondary infections are not significant while the vaccination coverage is under 25. This fact shows the importance of the random variable $R_{e 0}$ to fix an appropriate vaccination level.

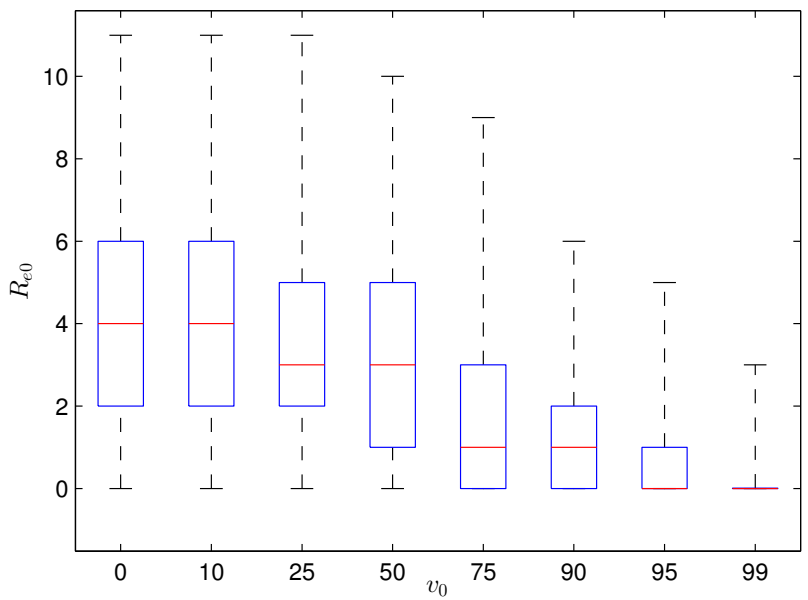

Fig. 11 Boxplot for $R_{e 0}$ under several vaccine coverage values when $N=100, \beta=10.0$, $\xi=0.1$ and $h=0.03$

Next we present some results relative to the global spread of the disease. In Figure 12, we compare $R_{c}, \bar{R}_{e 0}$ and $\bar{R}_{p}$ when the internal transmission rate $\beta$ varies over the interval $(0,20.0)$. As in previous scenarios, the recovery rate is $\gamma=1.0$. Vaccine coverage reaches half of the population and presents an effectiveness of $97 \%$. We compare results for populations of $N=100$ and 1000 individuals, with external transmission rate $\xi=1 / N$. For our parameter selection $R_{0}=\beta$ and $R_{c}=R_{0}(1-(1-h) / 2)=0.515 \beta$. Hence, as a function of $\beta$, the control reproduction number corresponds to the top line in Figure 
12. We notice that differences between $R_{c}$ and $R_{e 0}$ increase with increasing transmission rates. When $\beta=20.0$, we find $R_{c}=10.30$ and $\bar{R}_{e 0}=4.36$ for $N=100$, and $\bar{R}_{e 0}=5.67$ for $N=1000$. These magnitudes show the overestimation of the number of secondary cases of infection produced by the index case, as provided by $R_{c}$, and how the expected exact reproduction number $\bar{R}_{e 0}$ corrects the effect of the linearization hypothesis commonly assumed in the deterministic literature. Regarding the expected population transmission, $\bar{R}_{p}$, we conclude that it converges to the control reproduction number as the population size increases. In fact, for $N=1000$ differences between $R_{c}$ and $\bar{R}_{p}$ are smaller than 0.4 when $\beta \in(0,20.0)$. Hence, the line for $\bar{R}_{p}$ is graphically indistinguishable from $R_{c}$ and it is not plotted for $N=1000$.

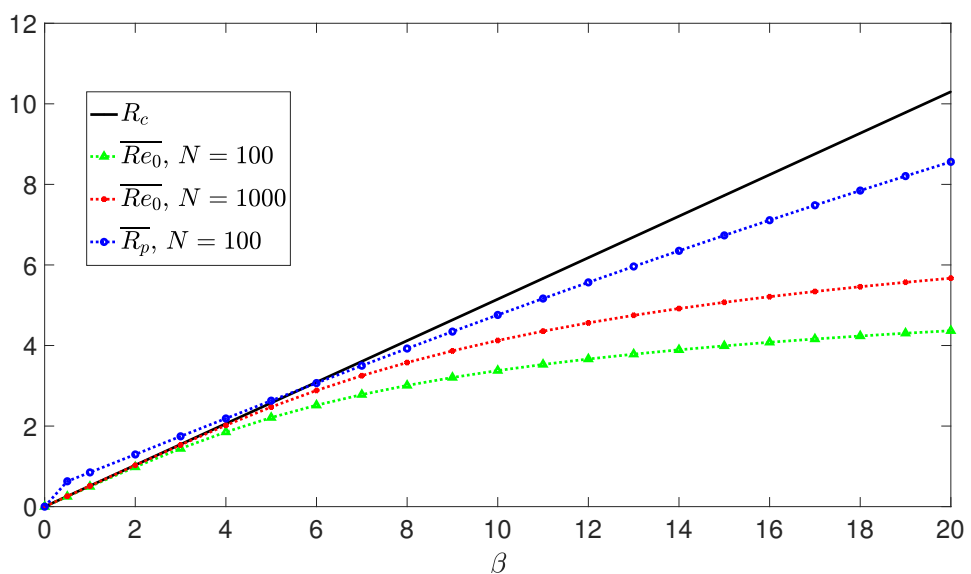

Fig. 12 Comparing $R_{c}, \bar{R}_{e 0}$ and $\bar{R}_{p}$ versus the internal transmission rate when $\xi=1 / N$, $h=0.03$ and $v_{0}=N / 2$

Finally, Table 3 shows the minimal vaccination coverage needed to interrupt epidemic transmission; that is, in order to get values smaller than one for the expected number of secondary cases coming from the index case. Notice that if the expected transmission (either from the whole set of infectious or limited to the index case) is less than 1, each infected individual transmits the disease to less than one person, which means that not every case will result in a new individual infection and epidemic transmission will cease. We display results for a population of $N=1000$ individuals, where the external transmission rate is taken as $\xi=1 / N$ and the internal rate $\beta$ is assumed to be at least 1 . We compare two scenarios by considering that the effectiveness of a vaccine is either $97 \%$ or $90 \%$. Values represented by $v_{c}$ give the herd-immunity threshold based on the control reproduction number $R_{c}$ (i.e., starting from $R_{0}=\beta / \gamma$, coverage is chosen as $v_{c} / N>\left(R_{0}-1\right) /\left(R_{0}(1-h)\right)$ in order that $\left.R_{c}<1\right)$. Additionally, $v_{e 0}$ and $v_{p}$ are minimal critical levels that guarantee that expected values $\bar{R}_{e 0}$ and $\bar{R}_{p}$, respectively, are less than one. 
Numerical values come from an iterative application of Algorithm 1 for $R_{e 0}$ and its variant for $R_{p}$, by raising initial coverage until expected values fulfill the required condition. Any dash symbol in Table 3 means that even a 100\% vaccine coverage does not guarantee that the expected number of secondary cases (measured in terms of $R_{c}, \bar{R}_{e 0}$ or $\bar{R}_{p}$ ) are less than one. Note that, as $\bar{R}_{p}$ includes also secondary cases arising from external infectious individuals, the minimal critical level $v_{p}$ presents higher values, at a fixed transmission rate $\beta$, when it is compared with $v_{c}$ or $v_{e 0}$. On the other hand, when the internal transmission rate $\beta$ increases, higher immunization coverage is needed to keep the expansion of the epidemic under control.

Table 3 Minimum level of vaccination with parameters $N=1000$ and $\xi=1 / N$

\begin{tabular}{|c|c|c|c|c|c|c|}
\hline & \multicolumn{3}{|c|}{$h=0.03$} & \multicolumn{3}{c|}{$h=0.1$} \\
\hline$\beta$ & $v_{c}$ & $v_{e 0}$ & $v_{p}$ & $v_{c}$ & $v_{e 0}$ & $v_{p}$ \\
\hline 1 & 0 & 0 & 413 & 0 & 0 & 445 \\
\hline 1.5 & 344 & 341 & 556 & 370 & 368 & 695 \\
\hline 2 & 515 & 513 & 645 & 556 & 554 & 808 \\
\hline 3 & 687 & 686 & 750 & 741 & 739 & 873 \\
\hline 4 & 773 & 772 & 809 & 833 & 832 & 915 \\
\hline 5 & 825 & 823 & 848 & 889 & 888 & 944 \\
\hline 6 & 859 & 858 & 875 & 926 & 925 & 966 \\
\hline 7 & 884 & 882 & 896 & 952 & 952 & 982 \\
\hline 8 & 902 & 902 & 911 & 972 & 972 & 996 \\
\hline 9 & 916 & 915 & 923 & 988 & 988 & - \\
\hline 10 & 928 & 927 & 933 & - & - & - \\
\hline 11 & 937 & 936 & 942 & - & - & - \\
\hline 12 & 945 & 944 & 949 & - & - & - \\
\hline
\end{tabular}

\section{Conclusions}

This paper studies infectious disease dynamics in a stochastic framework, where a Markov chain is used to model disease transmission. The continuous time Markov chain models changes in the state of the process defined as the number of individuals that are susceptible, infected or vaccinated. Assuming that susceptible and vaccinated individuals (due to vaccine failures) can get the infection through both internal and external contacts makes the model more realistic than those with only internal contacts.

Deterministic models are very useful in understanding the dynamics of infectious diseases and estimating important epidemiological descriptors as, for instance, the basic reproduction number. In this context, populations are relatively large, and the effect of depletion of susceptibles is minor but leads to reproductive numbers that overestimate the transmission potential of the disease. Our research involves a pair of random variables, $R_{e 0}$ and $R_{p}$, as alternative measures of the control reproduction number $R_{c}$. The stochastic 
Markovian framework allows us to identify the effective event leading to a new contagion. Consequently, we exclude repeated contacts established between the index case and already infected individuals, thus correcting for the effect of the linearization assumption commonly assumed in the deterministic framework and which produces the overestimation of the reproductive potential of a disease.

Concerning speed, our numerical algorithms provide results depending strongly on the population size and the other parameters of the model. For instance, the derivation of $\bar{R}_{e 0}$ through Algorithm 1 involves factorial moments $m_{v, i}^{1}$ of $\left(N-v_{0} / 2\right)\left(v_{0}+1\right)$ transient states, which for the particular choice of $N=1000$ and $v_{0}=N / 2$ implies 375,750 such states and requires $118 \mathrm{sec}$ CPU time, in a personal computer of $2.31 \mathrm{GHz}$ and $6 \mathrm{~GB}$ RAM.

Numerical results regarding vaccination coverage levels should be seen as a first approach to investigate optimal policies for controlling the spread of an infectious disease. The aim for future research is to develop health policies based on time horizons and warning levels related to the remaining vaccinated individuals.

This research can be generalized to different models for imperfect vaccine response by considering vaccines that reduce the probability of infection or that confer protection that wanes over time (see, for instance, Ball et al. 2008, Ball and Sirl 2018). Additionally, more sophisticated models could be treated by introducing population structures such as households, and also by including assumptions concerning to epidemics showing latent infectious periods or lifetime immunity after recovery.

\section{References}

1. Aguiar M, Martins J, Pinto A, Stollenwerk N (2010) Dynamics of epidemiological models. Acta Biotheor 58:381-389. https://doi.org/10.1007/s10441-010-9116-7

2. Alexander ME, Bowman C, Moghadas SM, Summers R, Gumel AB, Sahai BM (2004) A vaccination model for transmission dynamics of influenza. SIAM J Appl Dyn Syst 3:503524. https://doi.org/10.1137/030600370

3. Alexander ME, Moghadas SM, Rohani P, Summers AR (2006) Modelling the effect of a booster vaccination on disease epidemiology. J Math Biol 52:290-306. https://doi.org/10.1007/s00285-005-0356-0

4. Almaraz E, Gómez-Corral A (2018) On SIR-models with Markov-modulated events: Length of an outbreak, total size of the epidemic and number of secondary infections. AIMS 23:2153-2176 https://doi.org/10.3934/dcdsb.2018229

5. Almaraz E, Gómez-Corral A, Rodríguez-Bernal MT (2016) On the time to reach a critical number of infections in epidemic models with infective and susceptible immigrants. Biosystems 144:68-77. https://doi.org/10.1016/j.biosystems.2016.04.007

6. Amador J (2016) The SEIQS stochastic epidemic model with external source of infection. Appl Math Model 40:8352-8365. http://dx.doi.org/10.1016/j.apm.2016.04.023

7. Anderson M, Britton T (2000) Stochastic epidemics in dynamic populations: quasi-stationarity and extinction. J Math Bio 41: 559-580. https://doi.org/10.1007/s002850000060

8. Arino J, McCluskey CC, van den Driessche P (2003) Global results for an epidemic model with vaccination that exhibits backward bifurcation. SIAM J Appl Math 64:260276. https://epubs.siam.org/doi/abs/10.1137/S0036139902413829 
9. Arino J, Sun C, Yan W (2010) Global analysis for a general epidemiological model with vaccination and varying population. J Math Anal Appl 372:208223. https://doi.org/10.1016/j.jmaa.2010.07.017

10. Artalejo JR (2014) On the Markovian approach for modeling the dynamics of nosocomial infections. Acta Biotheor 62:15-34. https://doi.org/10.1007/s10441-013-9204-6

11. Artalejo JR, Lopez-Herrero MJ (2013) On the exact measure of disease spread in stochastic epidemic models. Bull Math Biol 75:1031-1050. https://doi.org/10.1007/s11538013-9836-3

12. Artalejo JR, Lopez-Herrero MJ (2014) Stochastic epidemic models: New behavioral indicators of the disease spreading. Appl Math Model 38:4371-4387. https://doi.org/10.1016/j.apm.2014.02.017

13. Ball F, Knock ES, O'Neill PD (2008) Control of emerging infectious diseases using responsive imperfect vaccination and isolation. Math Biosci 216:100-113. https://doi.org/10.1016/j.mbs.2008.08.008

14. Ball F, O'Neill PD, Pike J (2007) Stochastic epidemic models in structured populations featuring dynamic vaccination and isolation. J Appl Probab 44:571-585. https://doi.org/10.1239/jap/1189717530

15. Ball F, Sirl D (2013) Acquaintance vaccination in an epidemic on a random graph with specified degree distribution. J Appl Probab 50:1147-1168. https://doi.org/10.1239/jap/1389370105

16. Ball F, Sirl D (2018) Evaluation of vaccination strategies for SIR epidemics on random networks incorporating household structure. J Math Biol 76: 483-530. https://doi.org/10.1007/s00285-017-1139-0

17. Cheng Y, Pan Q, He M (2015) Psychological and behavioral effects in epidemiological model with imperfect vaccination compartment. Math Meth Appl Sci 38:4729-4740 https://doi.org/10.1002/mma.3387

18. Demicheli V, Rivetti A, Debalini MG, Di Pietrantonj C (2012) Vaccines for measles, mumps and rubella in children. Cochrane Database Syst Rev 2:CD004407.

19. Eckalbar JC, Eckalbar WL (2015) Dynamics of an SIR model with vaccination dependent on past prevalence with high-order distributed delay. Biosystems 129:50-65. https://doi.org/10.1016/j.biosystems.2014.12.004

20. Economou A, Gómez-Corral A, López-García M (2015) A stochastic SIS epidemic model with heterogeneous contacts. Physica A Stat Mech Appl 421:78-97. https://doi.org/10.1016/j.physa.2014.10.054

21. Golub GH and van Loan CF (1996) Matrix Computations. John Hopkins University Press, Baltimore

22. Gómez-Corral A, López-Garcia M (2015) Lifetime and reproduction of a marked individual in a two-species competition process. Appl Math Comput 264:223-245. https://doi.org/10.1016/j.amc.2015.04.061

23. Gómez-Corral A, López-Garcia M (2017) On SIR epidemic models with generally distributed infectious periods: Number of secondary cases and probability of infection. Internat J Biomath 10:1750024. https://doi.org/10.1142/S1793524517500243

24. Guo Y (2017) Stochastic regime switching SIS epidemic model with vaccination driven by Lévy noise. Adv Differential Equations 2017:375. https://doi.org/10.1186/s13662-0171424-7

25. Iannelli M, Martcheva M, Li XZ (2005) Strain replacement in an epidemic model with super-infection and perfect vaccination. Math Biosci 195:23-46. https://doi.org/10.1016/j.mbs.2005.01.004

26. Kermack WO, McKendrick AG (1927) Contribution to the mathematical theory of epidemics. Proc R Soc Lond A 115: 700-721. https://doi.org/10.1098/rspa.1927.0118

27. Keeling MJ, Ross JV (2008) On methods for studying stochastic disease dynamics. J R Soc Interface 5:171-181. https://doi.org/10.1098/rsif.2007.1106

28. Kretzschmar M, Welte R, van den Hoek A, Postma MJ (2001) Comparative model-based analysis of screening programs for Chlamydia trachomatis infections. Am J Epidemiol 153:90-101. https://doi.org/10.1093/aje/153.1.90

29. Kribs-Zaleta CM, Martcheva M (2002) Vaccination strategies and backward bifurcation in an age-since-infection structured model. Math Biosci 177:317-332. https://doi.org/10.1016/S0025-5564(01)00099-2 
30. Li W, Zhang Q (2019) Construction of positivity-preserving numerical method for stochastic SIVS epidemic model. Adv Difference Equ 219:25. https://doi.org/10.1186/s13662-019-1966-y

31. Lin Y, Jiang D, Wang S (2014) Stationary distribution of a stochastic SIS epidemic model with vaccination. Physica A 394:187-197. https://doi.org/10.1016/j.physa.2013.10.006

32. Liu Q, Jiang D, Shi N, Hayat T, Alsaedi A (2018) The threshold of a stochastic SIS epidemic model with imperfect vaccination. Math Computers in Simulation 144:78-90. https://doi.org/10.1016/j.matcom.2017.06.004

33. López-García M (2016) Stochastic descriptors in an SIR epidemic model for heterogeneous individuals in small networks. Math Biosci 271:42-61. https://doi.org/10.1016/j.mbs.2015.10.010

34. Lopez-Herrero MJ (2017) Epidemic transmission on SEIR stochastic models with nonlinear incidence rate. Math Method Appl Sci 40:2532-2541. https://doi.org/10.1002/mma.4179

35. Magpantay FM (2014) Epidemiological consequences of imperfect vaccines for immunizing infections. SIAM J Appl Math, 74:1810-1830. https://doi.org/10.1137/140956695

36. Marchette DJ, Wierman JC (2004) Modeling computer virus prevalence with a susceptible-infected-susceptible model with reintroduction. Comput Stat Data An 45:3-23. https://doi.org/10.1016/S0167-9473(03)00113-0

37. Moghadas SM (2004) Modelling the effect of imperfect vaccines on disease epidemiology. Discrete Continuous Dyn Syst Ser B 4:999-1012. https://doi.org/10.3934/dcdsb.2004.4.999

38. Ross JV (2011) Invasion of infectious diseases in finite homogeneous populations. J Theor Biol 289:83-89. https://doi.org/10.1016/j.jtbi.2011.08.035

39. Safan M, Rihan FA (2014) Mathematical analysis of an SIS model with imperfect vaccination and backward bifurcation. Math Comput Simulat 96:195-206. https://doi.org/10.1016/j.matcom.2011.07.007

40. Samanta GP (2015) Mathematical analysis of a Chlamydia epidemic model with pulse vaccination strategy. Acta Biotheor 63: 1-21. https://doi.org/10.1007/s10441-014-9234-8

41. Stone P, Wilkinson-Herbot H, Isham V (2007) A stochastic model for head lice infections. Math Biol 56:743-763. https://doi.org/10.1007/s00285-007-0136-0

42. Tijms HC (2003) A First Course in Stochastic Models. Wiley, Chichester.

43. Xiao Y, Tang S (2010) Dynamics of infection with nonlinear incidence in a simple vaccination model. Nonlinear Anal Real World Appl 11:4154-4163. https://doi.org/10.1016/j.nonrwa.2010.05.002

44. Yang J, Martcheva M, Wang L (2015) Global threshold dynamics on an SIVS model with waning vacine-induced immunity and nonlinear incidence. Math Biosci 268:1-8. https://doi.org/10.1016/j.mbs.2015.07.003 